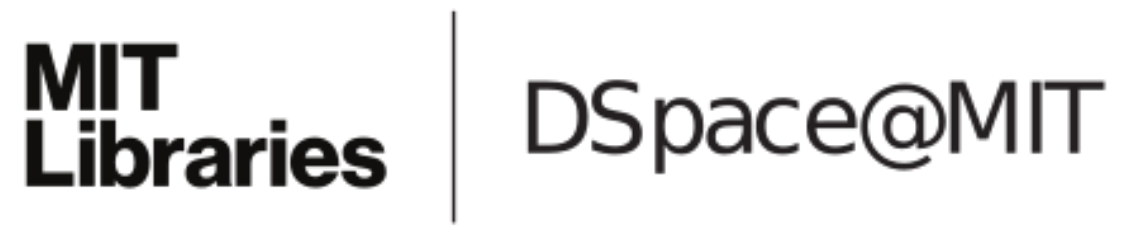

\author{
MIT Open Access Articles
}

Loops, sign structures, and emergent Fermi statistics in three-dimensional quantum dimer models

The MIT Faculty has made this article openly available. Please share how this access benefits you. Your story matters.

Citation: Ivanov, Vsevolod, Yang Qi, and Liang Fu. “Loops, Sign Structures, and Emergent Fermi Statistics in Three-Dimensional Quantum Dimer Models." Phys. Rev. B 89, no. 8 (February 2014). (C) 2014 American Physical Society

As Published: http://dx.doi.org/10.1103/PhysRevB.89.085128

Publisher: American Physical Society

Persistent URL: http://hdl.handle.net/1721.1/88760

Version: Final published version: final published article, as it appeared in a journal, conference proceedings, or other formally published context

Terms of Use: Article is made available in accordance with the publisher's policy and may be subject to US copyright law. Please refer to the publisher's site for terms of use. 


\title{
Loops, sign structures, and emergent Fermi statistics in three-dimensional quantum dimer models
}

\author{
Vsevolod Ivanov, ${ }^{1, *}$ Yang $\mathrm{Qi}^{2},{ }^{2}$ and Liang $\mathrm{Fu}^{1}$ \\ ${ }^{1}$ Department of Physics, Massachusetts Institute of Technology, Cambridge, Massachusetts 02139, USA \\ ${ }^{2}$ Institute of Advanced Study, Tsinghua University, Beijing 100084, China \\ (Received 6 October 2013; revised manuscript received 12 February 2014; published 26 February 2014)
}

\begin{abstract}
We introduce and study three-dimensional quantum dimer models with positive resonance terms. We demonstrate that their ground state wave functions exhibit a nonlocal sign structure that can be exactly formulated in terms of loops, and as a direct consequence, monomer excitations obey Fermi statistics. The sign structure and Fermi statistics in these "signful" quantum dimer models can be naturally described by a parton construction, which becomes exact at the solvable point.
\end{abstract}

DOI: 10.1103/PhysRevB.89.085128

PACS number(s): 75.10.Kt, 05.30.-d, 71.10.Pm

The Rokhsar-Kivelson (RK) quantum dimer model was originally introduced [1] to describe the short-range resonating-valence-bond (RVB) state in two dimensions [2]. Later it was discovered that quantum dimer models provide particularly simple and elegant realizations of topological phases of matter, including a two-dimensional (2D) gapped phase with $Z_{2}$ topological order [3], and a three-dimensional (3D) Coulomb phase described by an emergent Maxwell electrodynamics $[4,5]$. Both phases possess a fractional quasiparticle called a monomer, a deconfined pointlike excitation that carries half of the $U(1)$ charge of a dimer.

The statistics of monomers was a subject of considerable attention and debate in early studies [6,7] and of continuing interest recently [8]. It was eventually settled $[9,10]$ that the statistics of monomers in two-dimensional quantum dimer models cannot be assigned in a universal way, because statistics can be altered by attaching a $\pi$ flux (vison [11]) to a monomer. On the other hand, a boson cannot be changed into a fermion by $\pi$-flux binding in three dimensions, because particles and flux lines are objects of different dimensions. This leaves open the possibility of monomers with Fermi statistics in 3D quantum dimer models, which is the subject of this work. We note that Fermi statistics also arises in other 3D boson models with an emergent $Z_{2}$ gauge field [12,13].

We introduce and study quantum dimer models in 3D nonbipartite lattices with positive resonance terms, in contrast to terms with negative coefficients in the original RK model [1]. We find a "twisted" $Z_{2}$ topological phase in which monomers are deconfined and obey Fermi statistics. The Fermi statistics arises from a nonlocal sign structure of the ground state wave function, which is specified through loops in the transition graph by an exact sign rule. We provide a parton construction for these "signful" quantum dimer models, which yields the exact ground state dimer wave function and naturally explains the emergent Fermi statistics of monomers. Finally, we discuss the implications of our study for doped quantum dimer models and resonating-valence-bond (RVB) states in three dimensions.

While our main results generically apply to $3 \mathrm{D}$ quantum dimer models on non-bipartite lattices, we illustrate the essential physics using a 3D lattice made of corner-sharing octahedra (CSO), shown in Fig. 1(a). Each site is touched by

\footnotetext{
*Present address: Department of Physics, California Institute of Technology, Pasadena, California 91125, USA.
}

exactly one dimer occupying one of the eight nearest-neighbor bonds. The quantum dimer model Hamiltonian $H$ consists of resonance moves that locally flip a dimer configuration, and potential terms that give an energy cost for every flippable configuration.

For simplicity we only consider shortest resonance loops of length four. There exist two types of length-four resonance loops, having the shape of a square and a bent square, respectively [see Fig. 1(b)]. $H$ is thus given by

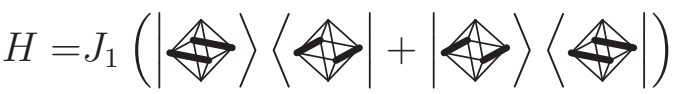

$$
\begin{aligned}
& +K_{1}(|\mathbb{A}\rangle\langle\mathbb{A}|+| \mathbb{\mathbb { V }}\rangle\langle\mathbb{\mathbb { }}|) \\
& +J_{2}(|\mathbb{W}\rangle\langle\mathbb{B}|+| \mathbb{W}\rangle\langle\mathbb{W}|) \\
& +K_{2}(|\mathbb{W}\rangle\langle\mathbb{W}|+| \mathbb{D}\rangle\langle\mathbb{D}|) \\
& +\cdots
\end{aligned}
$$

Here.. denotes similar terms for resonance loops of longer length, whose form will be discussed later.

When $\left|J_{i}\right|=K_{i}>0, H$ can be written as a sum of positive semidefinite projection operators:

$$
\begin{aligned}
& H=K_{1}\left(|\mathbb{\nabla}\rangle+\eta_{1}|\mathbb{\Downarrow}\rangle\right)\left(\langle\mathbb{\forall}|+\eta_{1}\langle\notin|\right)
\end{aligned}
$$

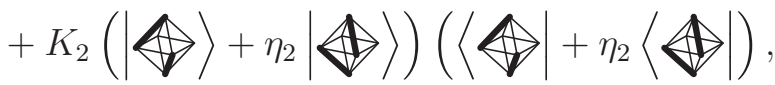

where $\eta_{i}=J_{i} / K_{i}= \pm 1 . \eta_{1}=\eta_{2}=-1$ corresponds to the original RK solvable point with negative resonance terms. In this case, the ground state is an equal amplitude superposition of all dimer coverings. Such a dimer-liquid state in a 3D CSO lattice is expected to represent a gapped $Z_{2}$ topological phase similar to the one in a face-centered-cubic lattice [5]. Since this RK wave function is everywhere positive, monomer excitations above the ground state are necessarily bosons.

Here we study quantum dimer models with positive resonance terms: $J_{1}, J_{2}>0$. In this case, ground states are "signful," taking both positive and negative values for different dimer coverings. Such a nontrivial sign structure is a 


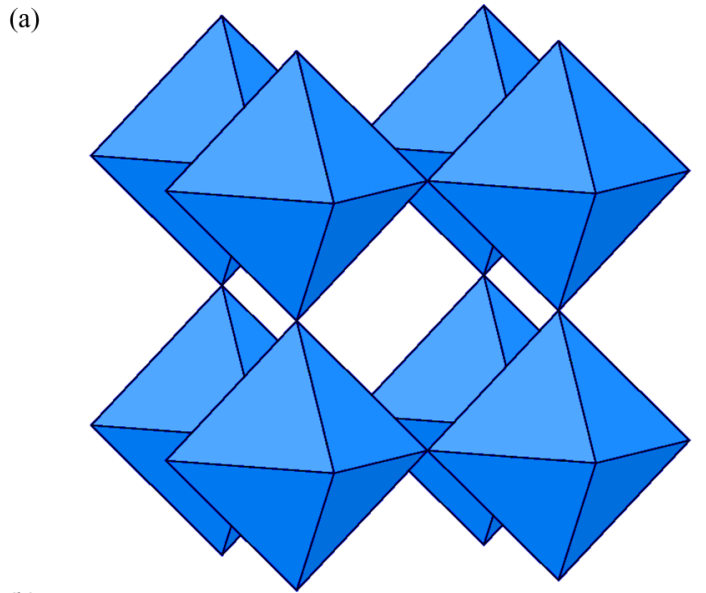

(b)

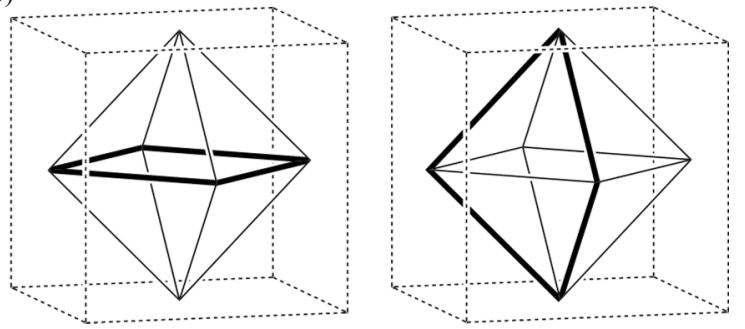

FIG. 1. (Color online) (a) Eight cubic unit cells of the CSO lattice. (b) The two types of length-4 loops on the CSO lattice. Left: Square loop, right: bent-square loop.

prerequisite to the emergence of fermionic monomer excitations. Following the spirit of the RK approach, we first construct a signful dimer wave function and then demonstrate that this wave function is the zero-energy ground state of $H$ at a generalized RK point $J_{i}=K_{i}>0$.

\section{LOOPS AND SIGN STRUCTURES}

The dimer wave function we constructed is an equal weight superposition of all dimer coverings $\left\{D_{a}\right\}$ with $a=1, \ldots, N_{D}$ with a sign structure:

$$
\Psi\left(D_{a}\right)=\frac{1}{\sqrt{N_{D}}} s\left(D_{a}\right) .
$$

The key ingredient for the emergent Fermi statistics is the form of $s\left(D_{a}\right)$, which we now define. First, we create a transition graph $G_{1 a}$ by superimposing $D_{a}$ and an arbitrary reference dimer covering $D_{1}$. The transition graph consists of nonintersecting even-length loops. Since dimer coverings and transition loops are in one-to-one correspondence, we define $s\left(D_{a}\right) \equiv s\left(G_{1 a}\right)$ as a function of transition loops.

The sign of transition loops is specified by endowing every nearest-neighbor bond with an arrow. The arrow pattern we choose for the CSO lattice is shown in Fig. 2. Now we define $s\left(G_{a b}\right)$ in terms of loops and arrows:

$$
s\left(G_{a b}\right)=(-1)^{N\left(G_{a b}\right)+W\left(G_{a b}\right)},
$$

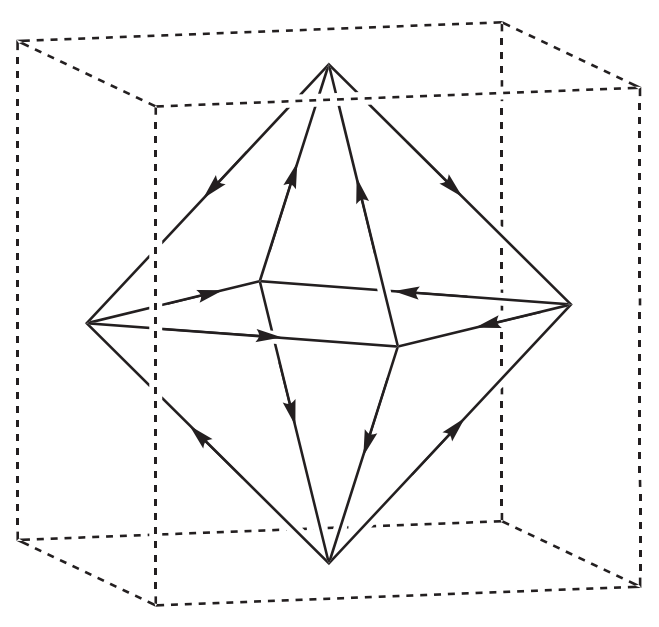

FIG. 2. Arrow pattern for CSO lattice.

where $N\left(G_{a b}\right)$ is the total number of loops in $G_{a b}$, and $W\left(G_{a b}\right)$ is the total number of "wrong-way" arrows when all loops are traversed unidirectionally.

The sign structure (4) we introduced for the quantum dimer wave function (3) is a central result of this work. It has several important properties. First, because all loops in the transition graph have an even length, $(-1)^{W\left(G_{a b}\right)}$ is independent of the direction of traverse, as it should be for loops in three dimensions. Second, $s\left(G_{a b}\right)$ is multiplicative under the composition of transition loops. For three different dimer coverings $D_{a}, D_{b}$, and $D_{c}$,

$$
s\left(G_{a c}\right)=s\left(G_{a b}\right) s\left(G_{b c}\right)
$$

This is obvious when $G_{a b}$ and $G_{b c}$ do not overlap, and is also true when they do. For example, when $G_{a b}$ and $G_{b c}$ overlap on one bond, two loops combine into one in $G_{a c}$, which reduces both the total number of loops and the number of wrong-way arrows by one. This result is proved for general cases in Appendix A and an alternative proof based on parton construction is given in Sec. III. Equation (5) guarantees that the dimer wave function defined using the sign structure $s\left(G_{a b}\right)$ does not depend on the choice of the reference dimer covering, up to an overall factor of -1 . Last but not the least, the arrow pattern in Fig. 2 guarantees that $\Psi\left(D_{a}\right)$ is invariant under all symmetry transformations of the CSO lattice, despite that the arrow pattern itself is not.

To justify the last point, we consider the symmetry transformation of the arrow pattern shown in Fig. 2. The symmetry of the CSO lattice can be represented by the $O_{h}$ point group, along with translation symmetry. We now check how the arrows transform under the symmetry operations of $O_{h}$, with +1 signifying that all arrows are unchanged, and -1 signifying that all arrows reverse:

\begin{tabular}{l|llllllllll}
$O_{h}$ & $E$ & $8 C_{3}$ & $6 C_{2}$ & $6 C_{4}$ & $3 C_{4}^{2}$ & $i$ & $6 S_{4}$ & $8 S_{6}$ & $3 \sigma_{h}$ & $6 \sigma_{d}$ \\
\hline$A_{2 g}$ & +1 & +1 & -1 & -1 & +1 & +1 & -1 & +1 & +1 & -1
\end{tabular}


This arrow pattern behaves as the representation $A_{2 g}$ of the $O_{h}$ point group. For a given transition graph, the sign of a particular loop given by Eq. (4) does not change under the transformation of reversing all arrows, as each loop contains an even number of dimers. Therefore the wave function defined in Eq. (3) is invariant under symmetry.

According to the arrow pattern, the signs $s\left(G_{a b}\right)$ of the two shortest-length transition loops, square and bent square, are -1 . It then follows that the dimer wave function $|\Psi\rangle$ is a zero-energy ground state of the quantum dimer model Hamiltonian (1) at the generalized RK point $J_{i}=K_{i}$. This completes our construction of solvable 3D quantum dimer models with a nontrivial sign structure.

It is worth pointing out that, as is common with quantum dimer models at the RK point, the Hamiltonian (1) has other zero-energy grounds states in addition to $\Psi\left(D_{a}\right)$, such as "dead" dimer coverings that are completely nonflippable. Also, similarly to quantum dimer models on other 3D lattices [14], we found that with only resonance terms on length-four loops the Hamiltonian does not connect all coverings in a given topological sector. By including additional resonance terms involving longer loops and choosing their signs according to the arrow pattern, one can increase the connectivity of dimer coverings, although the issue of ergodicity in $3 \mathrm{D}$ quantum dimer models is beyond the scope of this paper. On a positive side, we expect the dimer liquid state (3) to be stabilized in an extended regime $\left|J_{i}\right|>K_{i}$, as found for other non-bipartite lattices [3,5]. This issue can be studied numerically using Monte Carlo methods [14,15].

\section{STATISTICS OF MONOMERS}

We now demonstrate that the nontrivial sign structure of the ground state (4) directly gives rise to Fermi statistics of the monomer, a pointlike excitation associated with a site that is not touched by any dimer. Since a missing dimer can break up into two monomers, each monomer is a charge- $1 / 2$ fractional quasiparticle, which has a finite excitation energy associated with breaking the rule of one dimer per site.

Following the approach of Ref. [6], we determine the statistics by adiabatically transporting two monomers along a path which exchanges their positions, and examining the resulting change in the phase of the many-body wave function. To implement the exchange, we introduce dimer move terms $H_{T}$ into the quantum dimer model, which enables a monomer to hop by exchanging with a nearby dimer. Without losing generality we consider the following form of $H_{T}$,

$$
H_{T}=\lambda \sum(|\mathbb{\bigotimes}\rangle\langle\notin|+\text { H. с. }) \text {, }
$$

where the sum runs over all symmetry-related moves that transport a monomer to the nearest-neighbor site (see Fig. 3). Here we consider only nearest-neighbor hoppings, but a general proof of Fermi statistics for general hopping terms is given in Sec. III.

We assume that $\lambda$ is small and moves the monomer adiabatically. In the limit of $\lambda \rightarrow 0$, the eigenstates of the system are the dimer wave functions with monomers at fixed locations. Particularly the dimer wave function with a monomer at site $i$, denoted as $\left|\Psi_{i}\right\rangle$, is a superposition of dimer

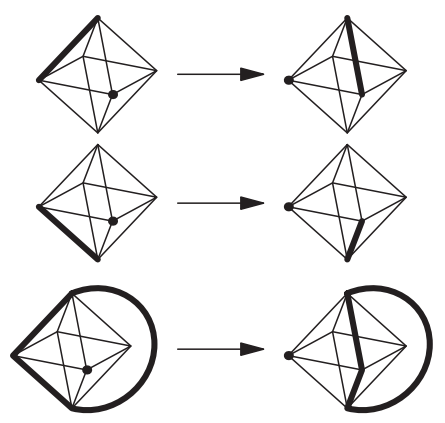

FIG. 3. Two different ways to move a monomer. The first and second rows show two different ways to move a monomer, and the last row shows the transition graph obtained by compositing the two dimer coverings in the same column. The curvy segment shows the part of the transition loop through the rest of the lattice, which is identical before and after the monomer move.

coverings that has no dimer connecting to site $i$, with the sign rule in Eq. (4) [16]. Under the adiabatic assumption, each time $H_{T}$ is applied to a monomer state to move a monomer from $i$ to $j$, it takes dimer coverings in $\left|\Psi_{i}\right\rangle$ and converts them to dimer coverings in $\left|\Psi_{j}\right\rangle$, and then the state of converted dimer coverings relaxes into the eigenstate $\left|\Psi_{j}\right\rangle$ before the monomer is moved again. Therefore the relative sign of the wave functions before and after the hopping is determined by projecting $H_{T}$ onto $\left|\Psi_{i}\right\rangle$ and $\left|\Psi_{j}\right\rangle$ as the following,

$$
t_{i j}=\left\langle\Psi_{j}\left|H_{T}\right| \Psi_{i}\right\rangle .
$$

There are different terms in $H_{T}$ that contribute to $t_{i j}$, which correspond to different ways to exchange a monomer and a dimer to move the former from $i$ to $j$, as illustrated in Fig. 3 . Here we show that these different terms contribute with the same sign to $t_{i j}$ in Eq. (7). In Fig. 3 it is illustrated that there are two ways to move a monomer from $i$ to $j$ by exchanging it with two different dimers, as shown on the first and second row. The relative phase between the two different dimer coverings in the initial and final states is determined by the transition loop on the bottom. The two transition loops in the initial and final states differ by moving two dimers from one side of a bent-square loop to the other side. Therefore the two transition loops have the same sign according to Eq. (4), as the arrow pattern shown in Fig. 2 has an even number of wrong-way arrows in any four-bond loop. This shows that different terms in Eq. (7) contribute to $t_{i j}$ with the same sign. Consequently the sign of $t_{i j}$ can be determined from any two dimer coverings in $\left|\Psi_{i}\right\rangle$ and $\left|\Psi_{j}\right\rangle$ that can be connected by $H_{T}$. Generally, this property holds for any arrow pattern with an even number of wrong-way arrows in four-bond loops, and a proof will be given in Sec. III.

Since quasiparticle statistics is path independent, we consider a simple process of exchanging two monomers at initial positions 1 and 3 via four nearest-neighbor monomer hops in the following sequence: $1 \rightarrow 2,2 \rightarrow 4,3 \rightarrow 1$, and $4 \rightarrow 3$ (see Fig. 4). After the exchange, the many-body state of the system comes back to itself, up to a possible phase change $\theta_{e x}$. Since the dimer wave functions are real, $\theta_{e x}$ is either 0 or $\pi . \theta_{e x}$ can be determined from the product of transition amplitudes 
(a)
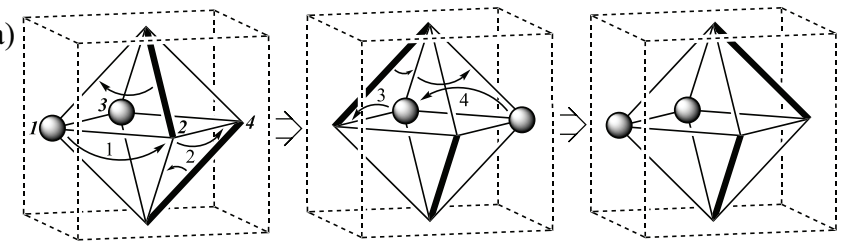

(b)
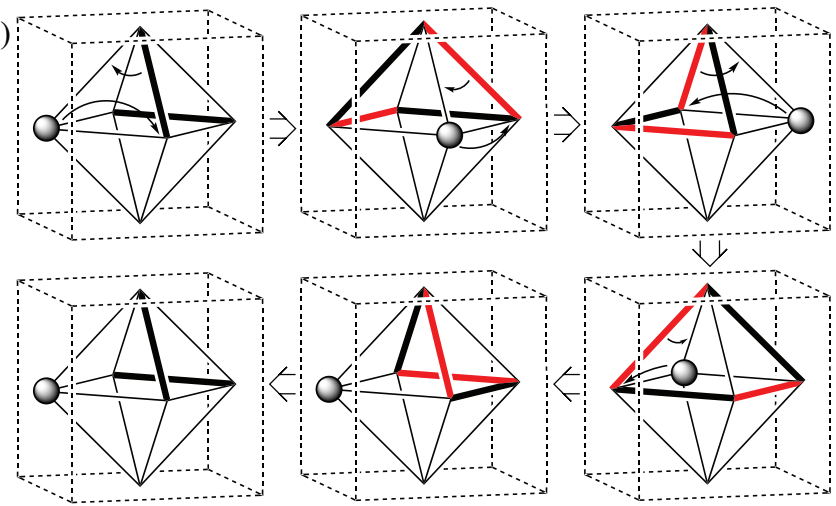

FIG. 4. (Color online) (a) The two holes are exchanged using nearest-neighbor hops defined by numbered arrows. Possible hole positions are marked in italics. Dimers are flipped accordingly. (b) We move a single hole along the same path to find the flux, changing our reference tiling using dimer flips along bent-square loops when necessary. When such a dimer flip is performed, the resulting new dimer tiling is marked in red. In total, 4 bent square loops are used, giving a +1 sign.

at every stage of the exchange process [12]

$$
t_{e x}=t_{12} t_{24} t_{31} t_{43} \text {. }
$$

The sign of $t_{e x}$ is unambiguous (independent of the gauge choice of dimer wave functions), and determines $\theta_{e x}: \theta_{e x}=0$ for $t_{e x}>0$ and $\pi$ for $t_{e x}<0$.

To evaluate (8), we use a natural sign convention for the many-body states $\left|\Psi_{i j}\right\rangle$ where $i, j$ label the positions of the two monomers, such that their amplitudes on the first four reference dimer coverings are positive:

$$
\Psi_{13}\left(D_{a}\right)=\Psi_{23}\left(D_{b}\right)=\Psi_{34}\left(D_{c}\right)=\Psi_{14}\left(D_{d}\right)>0,
$$

where the sequence of reference dimer coverings $D_{a} \rightarrow$ $D_{b} \rightarrow D_{c} \rightarrow D_{d} \rightarrow D_{e}$ are the ones that follow the sequence of dimer moves as illustrated in Fig. 4. Under this sign convention, $t_{12}, t_{24}, t_{31}$ have the same sign. Furthermore, since $D_{e}$ and $D_{a}$ only differ by a resonance loop on a bent square, the sign structure (4) dictates $\Psi_{13}\left(D_{e}\right)=-\Psi_{13}\left(D_{a}\right)$. Comparing with the convention $\Psi_{13}\left(D_{a}\right)>0$, this implies that $t_{43}$ have an opposite sign from the rest of the $t$ 's, which makes $t_{e x}$ negative. Therefore we conclude that the phase change after the monomer exchange is $\theta_{e x}=\pi$.

$\theta_{e x}$ is given by the sum of the statistical angle $\theta_{s}$, which is 0 for bosons and $\pi$ for fermions, and the background flux $\phi$ passing through the exchange paths of two particles that join into a loop $\Gamma$. Therefore to determine the quasiparticle statistics requires the knowledge of $\phi$ in addition to $\theta_{e x}$. For a real wave function, $\phi$ can only be 0 or $\pi$. $\phi$ can be determined from the phase change after transporting a single monomer around the loop $\Gamma$; here $\Gamma$ is the boundary of a single square plaquette.
Thus we now use the same set of dimer move operators $H_{T}$ and carry out a procedure similarly as before to compute $\phi$, with two important differences. First, the order in which the hopping terms are applied must change in order to form a continuous path, which is reflected by the new ordering of transition amplitudes:

$$
t_{e x}=t_{12} t_{24} t_{43} t_{31} .
$$

Second, each hole hop must be followed by a change in the reference dimer tiling, in order to guarantee that there exists a dimer-hole configuration that allows the hopping operation to be applied. The sequence of reference dimer tilings is $D_{a} \rightarrow$ $D_{b} \Rightarrow D_{b^{\prime}} \rightarrow D_{c} \Rightarrow D_{c^{\prime}} \rightarrow D_{d} \Rightarrow D_{d^{\prime}} \rightarrow D_{e} \Rightarrow D_{e^{\prime}}$, where $\rightarrow$ represents a hole hop while $\Rightarrow$ represents a change of reference. This sequence of operations is shown in Fig. 4(b), with each $D_{i^{\prime}}$ after a change of reference marked in red. We note that $D_{a}=D_{e^{\prime}}$, and that the final change of reference $D_{e} \Rightarrow D_{e^{\prime}}$ is carried out for the sake of convenience, since it allows us to refer to all transition loops as changes of reference. In this case, each change of reference corresponds to a bentsquare loop, and since we perform four such reference changes we find $\phi=0$. For clarity, we can write down the relative amplitudes of the many-body states $\left|\Psi_{i}\right\rangle$, where $i$ labels the positions of the monomer:

$$
\begin{aligned}
\Psi_{1}\left(D_{a}\right) & =\Psi_{2}\left(D_{b}\right)=-\Psi_{2}\left(D_{b^{\prime}}\right)=-\Psi_{4}\left(D_{c}\right)=\Psi_{4}\left(D_{c^{\prime}}\right) \\
& =\Psi_{3}\left(D_{d}\right)=-\Psi_{3}\left(D_{d^{\prime}}\right)=-\Psi_{1}\left(D_{e}\right)=\Psi_{1}\left(D_{e^{\prime}}\right),
\end{aligned}
$$

and we can see that in fact each reference change reverses the sign of the amplitude of the many-body state.

By combining the two results $\theta_{e x}=\pi$ and $\phi=0$, we conclude that monomers have a statistical angle $\theta_{s}=\pi$; i.e., they obey Fermi statistics. This conclusion is completely independent of how the two monomers exchanged. This is demonstrated with more examples in Appendix B, and can be proved for general cases using the parton wave function introduced in the next section.

\section{PARTON CONSTRUCTION}

In order to systemetically construct signful dimer wave functions with fermionic monomer excitations, we present a parton approach by writing a dimer degree of freedom in terms of two fermion variables:

$$
b_{i j}^{\dagger}=\xi_{i j} f_{i \alpha}^{\dagger} f_{j \beta}^{\dagger}, \quad \xi_{i j}=-\xi_{j i}= \pm 1,
$$

where $b_{i j}^{\dagger}$ is a hardcore boson operator that creates a dimer on a bond between two nearest-neighbor sites $i$ and $j$, and the fermion operators $f_{i \alpha}^{\dagger}$ and $f_{j \beta}^{\dagger}$ are defined at two new sites, which are located near the two ends $i$ and $j$ of the bond $i j$, respectively (see Fig. 5). These fermionic partons thus live on a decorated CSO lattice made of a 8-site cluster surrounding every site of the original lattice. The subscripts $\alpha$ and $\beta$ distinguish different sites within a cluster.

Due to the fermion anticommutation relation, we specify the ordering of fermion operators on the right-hand side of (10) using antisymmetric tensors $\xi_{i j}$ defined on all nearest-neighbor bonds, each of which takes two possible values \pm 1 . Specifying $\xi_{i j}$ on the bonds is equivalent to assigning the arrow pattern as discussed previously, and doing it consistently with lattice 
(a)

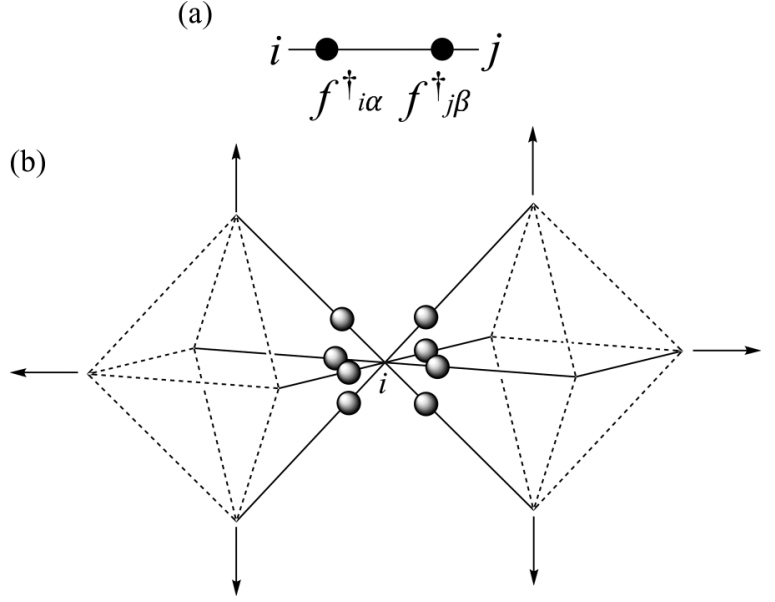

FIG. 5. Parton model. (a) One dimer degree of freedom is represented by two partons on the two ends of the dimer. (b) The partons live on a decorated CSO lattice in which every original CSO site is replaced by a eight-site cluster that represents the eight ways to draw a dimer from that site.

symmetries is central to our parton construction for quantum dimer models. For the CSO lattice here, we choose $\xi_{i j}$ 's according to the arrow pattern illustrated in Fig. 2: $\xi_{i j}=1$ if an arrow starts at $i$ and ends at $j$, and -1 if it is the opposite. In essence, the parton construction (10) treats a dimer as a composite of two fermions via the mapping between the Hilbert space of dimers and that of fermions $|n\rangle_{i j} \rightarrow|n\rangle_{i \alpha} \otimes$ $|n\rangle_{j \beta}$, where $n=0,1$ is the occupation number. However, the Hilbert space of fermions also includes unphysical states of the form $|n\rangle_{i \alpha} \otimes|1-n\rangle_{j \beta}$, which have no counterpart in the Hilbert space of dimers. Thus one must perform a Gutzwiller projection that removes all unphysical configurations within a parton wave function to generate a physical wave function.

A main advantage of the parton construction (10) is that simple wave functions for partons can yield highly nontrivial wave functions for dimers that are difficult to treat directly. Here we consider a very simple parton wave function that can reproduce the signful dimer wave function studied in Sec. I, and we shall discuss other possible extensions of parton wave functions as a way to construct variational dimer wave functions in Sec. IV. In this section we will focus on the following parton wave function,

$$
\left|\Phi_{f}\right\rangle=\prod_{i} \frac{1}{\sqrt{8}} \sum_{\alpha=1}^{8} f_{i \alpha}^{\dagger}|0\rangle
$$

which is a product of nonoverlapping "molecular" orbitals, one per cluster. For a given cluster, the molecular orbital has equal amplitudes on all 8 sites. We further obtain physical dimer wave function $|\Psi\rangle$ from $\left|\Phi_{f}\right\rangle$ :

$$
\Psi\left(i_{1} j_{1}, \ldots, i_{n} j_{n}\right)=\left\langle 0\left|\left(\prod_{i_{l} \alpha_{l}, j_{l} \beta_{l}} \xi_{i_{l} j_{l}} f_{i_{l} \alpha_{l}} f_{j_{l} \beta_{l}}\right)\right| \Phi_{f}\right\rangle,
$$

where $\Psi\left(i_{1} j_{1}, \ldots, i_{n} j_{n}\right)$ is the amplitude of having dimers on nearest-neighbor bonds $i_{1} j_{1}, \ldots, i_{n} j_{n}$.

The dimer wave function $|\Psi\rangle$ has several noteworthy properties that can be inferred straightforwardly from the parent parton wave function $\left|\Phi_{f}\right\rangle$. First, $|\Psi\rangle$ has exactly one dimer touching every lattice site. This is because (i) $\left|\Phi_{f}\right\rangle$ has exactly one parton per cluster, and (ii) two partons in adjacent clusters that share a bond must be simultaneously present to form a dimer. Second, since partons have a uniform density distribution in $\left|\Phi_{f}\right\rangle$, all possible dimer configurations appear with equal probability in $|\Psi\rangle$; i.e., $\left|\Psi\left(i_{1} j_{1}, \ldots, i_{n} j_{n}\right)\right|=\frac{1}{\sqrt{N_{D}}}$. Last but most importantly, due to the fermion nature of partons, the sign of $\Psi\left(i_{1} j_{1}, \ldots, i_{n} j_{n}\right)$ is not all positive but depends nontrivially on the dimer coverings following the same sign rule as stated in Eq. (4).

To obtain the nontrivial sign structure of $|\Psi\rangle$, we compare the relative sign of $\Psi\left(D_{1}\right)$ and $\Psi\left(D_{2}\right)$, where $D_{1}$ and $D_{2}$ are two arbitrary dimer configurations. We claim that the relative sign is determined from the transition graph $G_{12}$ as the following,

$$
\frac{\Psi\left(D_{1}\right)}{\Psi\left(D_{2}\right)}=\prod_{m}(-1)^{1+W\left(L_{m}\right)}=(-1)^{N\left(G_{12}\right)+W\left(G_{12}\right)},
$$

where $W\left(L_{m}\right)$ is the number of "wrong-way" arrows in the loop $L_{m}$, and $N\left(G_{12}\right)$ and $W\left(G_{12}\right)$ are the same functions counting number of loops and "wrong-way" arrows that were used in Eq. (4). To prove (13), it suffices to consider a single loop made of a sequence of lattice sites: $r_{1} \rightarrow r_{2} \rightarrow \cdots \rightarrow r_{2 k}$. The two corresponding dimer configurations are then $D_{1}=$ $\left(r_{1} r_{2}\right), \ldots,\left(r_{2 k-1} r_{2 k}\right)$ and $D_{2}=\left(r_{2 k} r_{1}\right), \ldots,\left(r_{2 k-2} r_{2 k-1}\right)$. It follows from (11) and (12) that $\Psi\left(D_{1}\right)$ and $\Psi\left(D_{2}\right)$ are respectively given by

$$
\begin{aligned}
& \Psi\left(D_{1}\right)=\xi_{r_{1} r_{2}} \cdots \xi_{r_{2 k-1} r_{2 k}}\left\langle 0\left|f_{r_{1}} f_{r_{2}} \ldots f_{r_{2 k-1}} f_{r_{2 k}}\right| \Phi_{f}\right\rangle, \\
& \Psi\left(D_{2}\right)=\xi_{r_{2 k} r_{1}} \cdots \xi_{r_{2 k-2} r_{2 k-1}}\left\langle 0\left|f_{r_{2 k}} f_{r_{1}} \ldots f_{r_{2 k-2}} f_{r_{2 k-1}}\right| \Phi_{f}\right\rangle .
\end{aligned}
$$

The ratio is thus given by

$$
\frac{\Psi\left(D_{1}\right)}{\Psi\left(D_{2}\right)}=-\prod_{i=1}^{2 n} \xi_{r_{i} r_{i+1}}, \quad r_{2 n+1} \equiv r_{1},
$$

where we have used the fermion anticommutation relation. This proves the sign rule (13).

Comparing the sign structures in Eq. (4) and Eq. (13), one can see that the wave function defined in Eq. (3) for a certain arrow configuration is the same as the parton wave function in Eq. (12) using the corresponding $\xi_{i j}$ assignment. Therefore the parton approach gives a systematic way to construct dimer wave functions with relative signs between different dimer configurations. This construction also provides an explicit proof of Eq. (5).

Furthermore, the parton approach gives a straightforward way to demonstrate that the monomer excitations carry Fermi statistics. To see this, we again study the phase difference between exchanging two holes and moving one hole along the same path as we did in the previous section, with the help of the reference parton wave function. For each step, a monomer is moved by flipping one dimer. Particularly, assume that a monomer is moved from site $i$ to site $j$ by moving one dimer from $(j k)$ to $(i k)$. Correspondingly, in the parton wave function one moves a hole with the following fermion hopping term,

$$
H_{T}^{f}=\sum_{i j} t_{i j}^{f} f_{j} f_{i}^{\dagger}
$$


where $f_{i}=\frac{1}{\sqrt{8}} \sum_{\alpha=1}^{8} f_{i \alpha}$, and $t_{i j}^{f}= \pm 1$ determines the relative sign between the wave functions before and after hopping. Here $t_{i j}^{f}$ is chosen such that the fermion hopping term $H_{T}^{f}$ has the same sign as the dimer hopping term $H_{T}$ defined in Eq. (6). According to the relation between dimer wave function and parton wave function in Eq. (12), the relative phase of the physical wave function before and after the projection is

$$
\frac{\Psi((i k) \cdots)}{\Psi((j k) \cdots)}=\frac{\left\langle 0\left|\xi_{i k} f_{i \alpha} f_{k \delta} t_{i j}^{f} f_{j} f_{i}^{\dagger} f_{k}^{\dagger} f_{j}^{\dagger}\right| 0\right\rangle}{\left\langle 0\left|\xi_{j k} f_{j \beta} f_{k \gamma} f_{k}^{\dagger} f_{j}^{\dagger}\right| 0\right\rangle}=\xi_{i k} \xi_{k j} t_{i j}^{f} .
$$

Here the left-hand side of the equation has the same sign as the corresponding term in Eq. (7). Hence we choose the sign of $t_{i j}^{f}$ such that the left-hand side has the sign of $t_{i j}$. This is achieved by choosing

$$
t_{i j}^{f}=\xi_{i k} \xi_{k j} \operatorname{sgn} t_{i j}
$$

With the condition in Eq. (17) satisfied, after each step of monomer hopping and dimer resonance, the sign of the dimer wave function is always determined from the corresponding parton wave function using the projection in Eq. (12). Hence the monomer excitation in the dimer wave function has the same Fermi statistics as the hole in the parton wave function.

It is important to note that the fermion hopping term in the parton construction only depends on the beginning and ending sites $i$ and $j$, while the monomer hopping from $i$ to $j$ is meditated by a dimer move $(j k) \rightarrow(i k)$. For lattices with high symmetry, a monomer hop from $i, j$ can be assisted by more than one such dimer movers that are symmetry-related to each other, as shown in Fig. 3 for the CSO lattice. In this case, it is crucial that the coefficients of $t_{i j}^{f}$ determined by Eq. (17) do not depend on the choice of possible dimer moves specified by $k$. We find that this is indeed the case for the CSO lattice, thanks to the arrow pattern specified in Fig. 2. This is because of the aforementioned fact that any length-four loop has an even number of wrong-way arrows. In other words, for any four points on a loop $i \rightarrow k \rightarrow j \rightarrow k^{\prime} \rightarrow i$, we have $\xi_{i k} \xi_{k j}=\xi_{i k^{\prime}} \xi_{k^{\prime} j}$, and therefore they give the same $t_{i j}^{f}$. This also implies that with a given $t_{i j}^{f}$, different ways of exchanging the monomer and a dimer contribute to $t_{i j}$ with the same sign. Hence this provides a general proof of this claim that was first introduced in Sec. II. Here our argument depends only on the fact that the arrow pattern has an even number of wrong-way arrows for all length-four loops, and can be generalized to other lattices where such arrow pattern can be assigned.

\section{DISCUSSION}

Using the CSO lattice as an example, we study a class of 3D quantum dimer models with frustrated resonance terms. We construct an exact ground state wave function of the model as a superposition of dimer configurations with a twisted sign structure. The monomer excitations in such a state are deconfined fermions. Furthermore, we give a systematic approach to construct such states using parton projective wave functions, and such construction naturally explains the Fermi statistics of the monomer excitations.
The dimer model studied in this work can potentially be realized in spin systems in a short-range RVB state where the spin-triplet gap is much larger than the spin-singlet gap [1]. In such a state, the spins are paired into spin-singlet valence bonds, and the ground state is a superposition of different valence-bond configurations. Such a state can be mapped to a dimer model by mapping spin valence bonds to dimers. In the RVB state, a valence bond can be broken and a spin-triplet excitation is created, similarly to the way a dimer breaks up into two monomers in a quantum dimer model. Therefore the monomer excitations in the dimer model can be viewed as spinon excitations in the RVB state. The difference between a monomer and a spinon, that the former carries a $U(1)$ quantum number but the latter carries an $S U(2)$ quantum number, can be eliminated by applying a Zeeman field to the RVB state. Therefore our signful wave function (3) can be used to describe a class of 3D spin liquids with fermionic spinon excitations. It is interesting to note that antiferromagnetic interactions between spins naturally lead to positive resonance terms in quantum dimer models studied in this work [1].

One can extend our wave function to study dimer models with finite density of monomers, which can be induced by applying a Zeeman field larger than the spin-triplet gap to the corresponding RVB state. Because monomers are fermions, the resulting state is likely to realize a Bose metal state [17], in which monomers form a Fermi sea and boson correlation functions exhibit Fermi-surface-like singularities. Using the parton construction presented in Sec. III, one can construct a variational wave function for the doped dimer models by projecting a parton wave function with a parton Fermi surface. Such wave functions can be studied numerically using the variational Monte Carlo method [18].

One can further regard the above RVB state as a description of an electronic system at half filling. Such a system will host spinless holon excitations in addition to chargeless spinons. The Fermi statistics of spinons then implies Bose statistics of holons. Doping this system away from half filling can induce holon condensation and leads to superconductivity [1,2]. However, unlike the original quantum dimer model, the superconducting state of a doped quantum dimer model studied in this work will have $d$-wave pairing symmetry. We shall leave these interesting extensions of our model to future studies.

\section{ACKNOWLEDGMENTS}

We thank Senthil Todadri and Jeongwan Haah for invaluable discussions. Y.Q. is supported by NSFC Grant No. 11104154. L.F. is supported by the DOE Office of Basic Energy Sciences, Division of Materials Sciences and Engineering, under Award No. DE-SC0010526. We thank the hospitality of Tsinghua University during the LT26 Satellite conference "Topological Insulators and Superconductors," when this work was initiated.

\section{APPENDIX A: PROOF OF MULTIPLICITY OF TRANSITION GRAPH SIGNS UNDER COMPOSITION}

In this appendix we discuss the relation between the signs of transition graphs and the sign of their composition, as described in Eq. (5). In the main text we see that this equation 


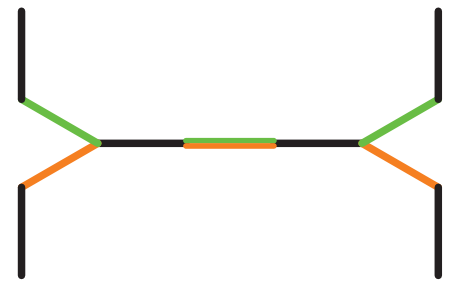

FIG. 6. (Color online) Overlapping boundary of two transition graphs. The black bonds belong to dimer covering $D_{b}$, green and orange bonds belong to dimer coverings $D_{a}$ and $D_{c}$, respectively, and the bond painted in both green and orange belongs to both $D_{a}$ and $D_{c}$.

holds when loops in $G_{a b}$ and $G_{b c}$ do not overlap or overlap on one bond. In this appendix we give a general proof for this equation. Before going through the proof we first note that according to the definition of $s\left(G_{a b}\right)$ in Eq. (4), length-two loops which are just two overlapping dimers always contribute a factor of +1 , so we can ignore them and only count nontrivial loops in Eq. (4).

When two transition graphs $G_{a b}$ and $G_{b c}$ are composited together, the transition loops may overlap on several pieces of overlapping boundaries. The resulting graph $G_{a c}$ is obtained by merging the loops at these overlapping boundaries. To prove Eq. (5) it is sufficient to show that the sign of the transition graph does not change before and after merging one piece of overlapping boundary.

First, we argue that each piece of overlapping boundary contains an odd number of bonds. As shown in Fig. 6, a piece of overlapping boundary must start and end on a bond in dimer covering $D_{b}$, since for any bond on the boundary that belongs to coverings $D_{a}$ and $D_{c}$, the bond adjacent to it belongs to $D_{b}$ and is thus present in both $G_{a b}$ and $G_{b c}$ and also belongs to the overlapping boundary. Between the two endings that belong to $D_{b}$, the boundary consists of alternating bonds from $D_{a} / D_{c}$ and from $D_{b}$ respectively. Therefore the total number of bonds in an overlapping boundary must be odd.

When merging a piece of overlapping boundary between two different loops, the two loops can be oriented such that they travel through the overlapping boundary in opposite directions (see Fig. 7), and after the merging the two loops are combined into one that inherits the orientation of the two original loops. After this merging the total number of loops is reduced by one, and the number of wrong-way arrows is reduced by the length of the boundary, which is an odd number, since each bond in

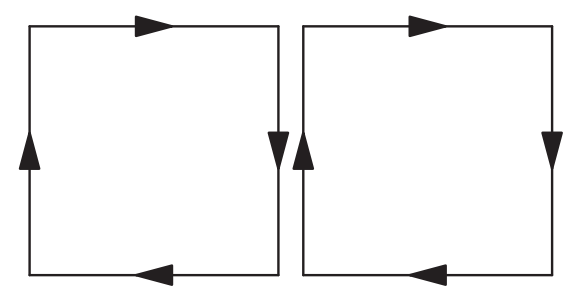

FIG. 7. Two loops with one overlapping boundary. The orientations of the two loops are chosen such that the boundaries are oriented in opposite directions on the two loops, and the merged loop can naturally inherit the orientations.

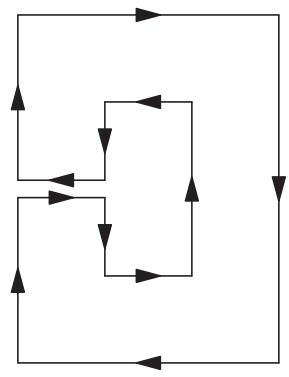

(a)

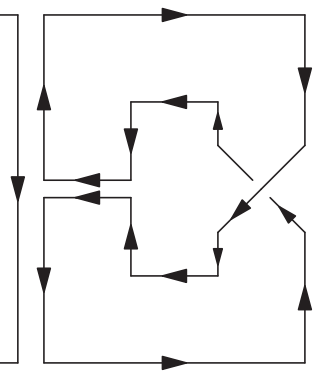

(b)

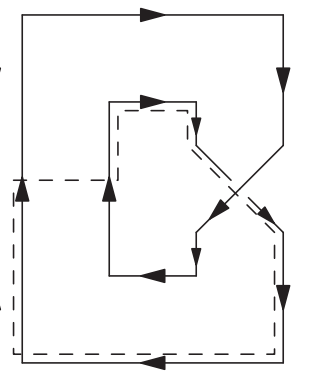

(c)
FIG. 8. One loop with one overlapping boundary. The arrow shows the orientation of the loop. (a) A planar loop without twisting. (b) A nonplanar loop with a twist. The loop is the edge of a Möbius strip. (c) The result of merging of the loop in (b). Comparing to the orientation in (b), the direction of the bonds labeled by the dashed line are flipped.

the boundary is traversed twice in two different directions in the original transition graphs. Consequently according to the sign rule in Eq. (4) the sign of the graph does not change before and after the merging.

On the other hand, when the piece of overlapping boundary belongs to the same loop (this happens when two loops overlap at more than one places: when merging the first piece of overlapping boundary it is the case shown in Fig. 7, and all subsequential merges are merging of the same loop), there are two possibilities: first, if the loop is planar, as shown in Fig. 8(a), after the merging the loop becomes two loops, and the number of loops is increased by one. Similarly to the previous case, one can arbitrarily fix the orientation of the loop and the overlapping boundary is traversed twice in opposite directions. Hence the number of wrong-way arrows is again reduced by an odd number. So the total sign stays the same.

The second case, which only exists in dimensions higher than two, is when the loop is twisted and forms the boundary of a Möbius strip, as shown in Fig. 8(b). In this case after the merging there is still one loop. Moreover, if we fix the orientations of the old and the new loop, the orientation needs to be flipped on one portion of the loop at the merging. The portion where the orientation is flipped, labeled by the dashed line in Fig. 8(c), also forms a transition loop itself and therefore contains an even number of bonds. Consequently the number of wrong-way arrows is changed by an even number. Combined with the invariant loop count this implies that the sign stays the same.

\section{APPENDIX B: CALCULATION OF MONOMER STATISTICS FOR VARIOUS EXCHANGES}

In this appendix we will further demonstrate the Fermi statistics of monomers by calculating the phase change $\theta_{e x}$ and background flux $\phi$ for various exchanges. We will determine these signs by considering the transition loops necessary to perform reference changes during each exchange using the procedure developed in Sec. II.

In Sec. II, we showed an exchange which only used nearestneighbor hops. We will now exchange two holes using only next-nearest-neighbor hops on the square lattice [Fig. 9(a)], 
(a)

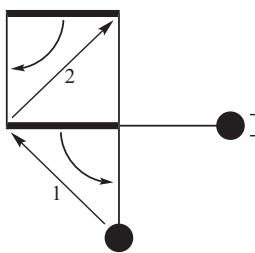

(b)
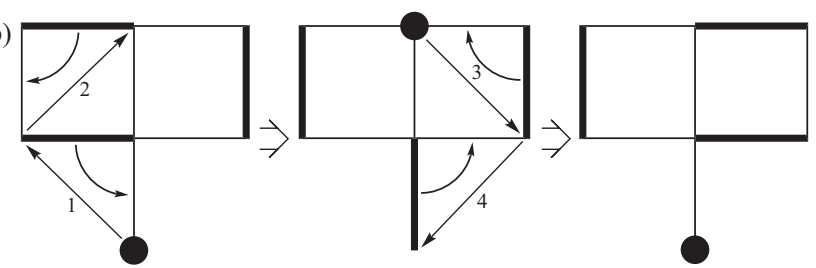

FIG. 9. (a) The two holes are exchanged on the square sublattice using next-nearest-neighbor hops, which requires only one square loop. (b) We move a single hole along the same path to find the background flux. Two square loops are needed, giving $\phi=0$.

which can be obtained by taking the subset of lattice sites that lie on a plane bisecting a layer of CSO unit cells. This exchange requires only one change of reference at the very end to return to the initial dimer tiling, which uses a transition graph with a single square loop, yielding $\theta_{e x}=\pi$. In order to calculate the statistical angle $\theta_{s}$, we need to find the flux $\phi$ for a single hole moving around the same path, using a different ordering of the hopping terms, along with new corresponding reference changes [Fig. 9(b)]. This time, there are two reference changes, each using a square loop. Since hopping terms do not affect the relative signs of the many-body states in the process, we only need to find the effect of the reference changes on the sign. For a general process with many different reference changes, we calculate the signs of each loop involved individually using Eq. (4), and then determine the overall sign by taking the product of all the individual signs of the loop using Eq. (5).

(a)

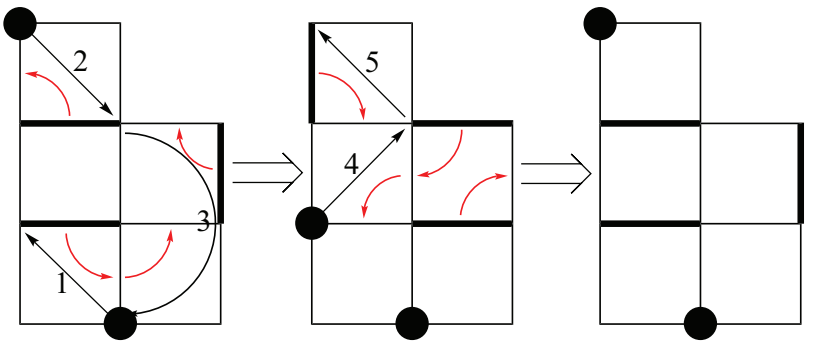

(b)

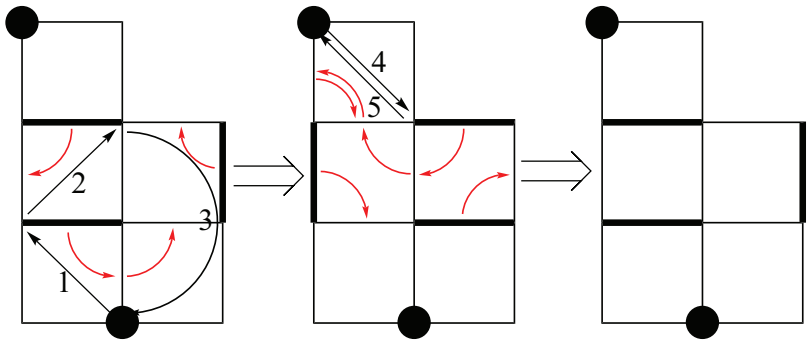

FIG. 10. (Color online) (a) A Levin-Wen exchange is performed on two holes. (b) We move a single hole along the same path to find that background flux $\phi=0$. (a)
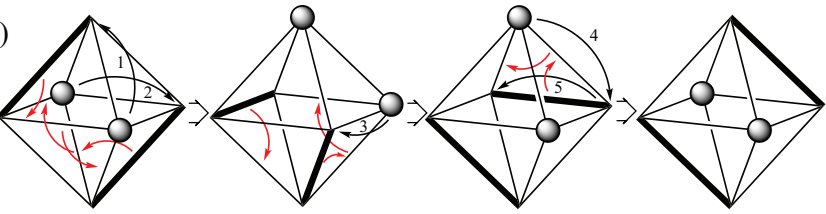

(b)
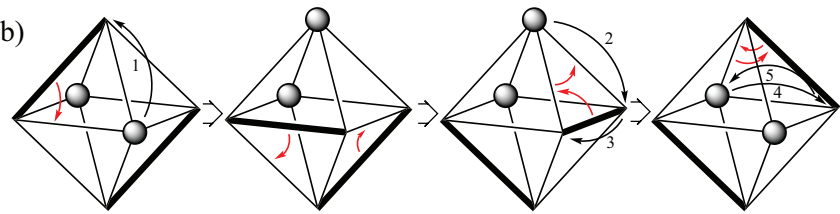

FIG. 11. (Color online) (a) A Levin-Wen exchange is performed on two holes. Two reference changes occur after the second and third hops. (b) The sequence of hops on two holes is repeated, this time without exchange. Only a single reference change is necessary, after the first hop.

For this particular case, two square loops give us an overall sign of +1 , meaning $\phi=0$. Therefore, $\theta_{s}=\theta_{e x}-\phi=\pi$.

We can perform a similar calculation using the five-step Levin-Wen exchange described in Ref. [12]. This approach sometimes has the advantage of producing more convenient exchanges, and can be used to calculate both $\theta_{e x}$ and $\phi$ using the same two monomers by performing a process of exchange and nonexchange, respectively, which will depend on the order of hopping terms (Fig. 10). In this case, the exchange process uses a single square loop, while the nonexchange process uses two square loops, which gives $\theta_{e x}=\pi, \phi=0, \theta_{s}=\pi$.

For completeness, we now present a similar Levin-Wen exchange using only nearest-neighbor hops (Fig. 11). Here we change reference twice, using a bent-square loop each time, while the initial and final dimer tilings are connected by a single square loop. Therefore the exchange angle is $\theta_{e x}=\pi$. For the nonexchange process, we need one bent-square loop for a change of reference, and one square loop to connect initial and final dimer tilings, which give a background flux of $\phi=$ 0 , so $\theta_{s}=\pi$.

So far, we have considered exchanges which have used only one type of hole-hopping term, and all exchanges have zero background flux. We now present an exchange which uses both nearest-neighbor and next-nearest-neighbor hopping

(a)
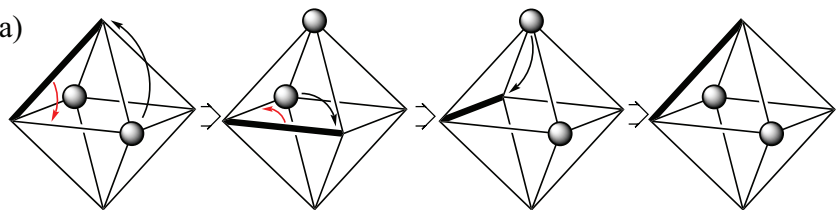

(b)
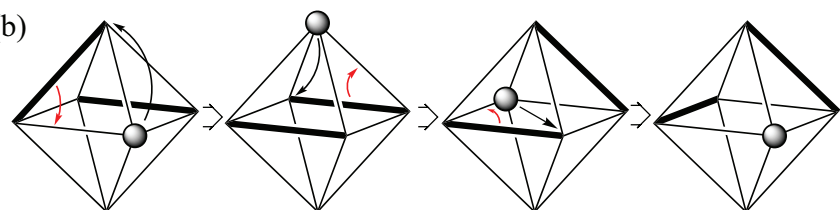

FIG. 12. (Color online) (a) Two holes are exchanged using two nearest-neighbor hops, and one next-nearest neighbor hop. Red arrows show corresponding dimer moves. (b) A single hole is moved along the same path, generating nonzero flux. 
and has nonzero background flux. It also has the interesting property that the exchange itself does not change the reference configuration. It is trivial to calculate that $\theta_{e x}=0$, since initial and final dimer tilings are identical, and no reference changes are needed (Fig. 12). When moving a single hole on the same path we require one bent-square loop, meaning that the background flux is nonzero: $\phi=\pi$. In this case, however, the exchange angle was 0 , so the statistical angle $\theta_{s}$ is still $\pi$.

These various examples demonstrate the persistence of Fermi statistics of monomer holes on the CSO lattice, regardless of the type of hopping terms included in the Hamiltonian, or the presence of background flux.
[1] D. S. Rokhsar and S. A. Kivelson, Phys. Rev. Lett. 61, 2376 (1988).

[2] P. W. Anderson, Science 235, 1196 (1987).

[3] R. Moessner and S. L. Sondhi, Phys. Rev. Lett. 86, 1881 (2001).

[4] M. Hermele, M. P. A. Fisher, and L. Balents, Phys. Rev. B 69, 064404 (2004).

[5] D. A. Huse, W. Krauth, R. Moessner, and S. L. Sondhi, Phys. Rev. Lett. 91, 167004 (2003).

[6] S. A. Kivelson, D. S. Rokhsar, and J. P. Sethna, Phys. Rev. B 35, 8865 (1987).

[7] F. D. M. Haldane and H. Levine, Phys. Rev. B 40, 7340 (1989).

[8] C. A. Lamas, A. Ralko, M. Oshikawa, D. Poilblanc, and P. Pujol, Phys. Rev. B 87, 104512 (2013).

[9] S. A. Kivelson, Phys. Rev. B 39, 259 (1989).

[10] N. Read and B. Chakraborty, Phys. Rev. B 40, 7133 (1989).

[11] T. Senthil and M. P. A. Fisher, Phys. Rev. B 62, 7850 (2000).
[12] M. Levin and X. G. Wen, Phys. Rev. B 67, 245316 (2003).

[13] I. Kimchi, J. G. Analytis, and A. Vishwanath, arXiv:1309.1171.

[14] O. Sikora, N. Shannon, F. Pollmann, K. Penc, and P. Fulde, Phys. Rev. B 84, 115129 (2011).

[15] The model with $J_{i}>0$ can be studied with the quantum Monte Carlo method without the sign problem after a nonlocal unitary transformation that changes the sign of the dimer basis using the factor $s(G)$ defined in Eq. (4), which maps the model to the one with $J_{i}<0$.

[16] Since monomers are fractionalized excitations, there must be an even number of monomers to form a valid dimer configuration, and here in the single-monomer wave function $\left|\Psi_{i}\right\rangle$ we assume there is another monomer fixed at a location far away from $i$.

[17] O. I. Motrunich and M. P. A. Fisher, Phys. Rev. B 75, 235116 (2007); M. Levin and T. Senthil, ibid. 78, 245111 (2008).

[18] C. Gros, Ann. Phys. 189, 53 (1989). 